\title{
Channel Feedback Codebook Design for Millimeter-Wave Massive MIMO Systems Relying on Lens Antenna Array
}

\author{
Wenqian Shen, Linglong Dai, Yue Li, Zhaocheng Wang, and Lajos Hanzo
}

\begin{abstract}
The recently proposed millimeter-wave (mmWave) massive MIMO system relying on a lens antenna array (LAA) significantly reduces the number of radio frequency $(\mathrm{RF})$ chains using beam selection. A high data rate can be achieved based on the reduced-dimensional equivalent channel after beam selection. In frequency division duplexing (FDD) systems, the equivalent channel has to be signaled back to the base station (BS) via a feedback channel based on a codebook. However, no dedicated codebook has been proposed for LAA-aided mm-Wave systems. To fill this gap, in this paper, we propose a reduced-dimensional subspace codebook (RDSC) for LAA-aided mmWave massive MIMO systems. Specifically, under the recently proposed concept of angle coherence time, we first generate the large-dimensional vectors in the channel subspace, which is determined by the angles-of-departure (AoDs) of the dominant paths. Then, based on these vectors in the channel subspace, we create the RDSC by considering both the lens and the beam selector. Finally, the equivalent channel is quantized using the proposed RDSC and fed back to the BS. Finally, we carry out mathematical performance analysis of the proposed RDSC and show that its feedback overhead is rendered proportional to the relatively small number of dominant paths per user. The analytical results are verified by our simulations.
\end{abstract}

Index Terms-MmWave massive MIMO, lens, FDD, channel feedback, codebook.

\section{INTRODUCTION}

$\mathbf{M}$ ILLIMETER-wave (mmWave) massive multiple-input multiple-output (MIMO) solutions are expected to find their way into next-generation wireless systems owing to their substantial bandwidth potential [1]. However, given the high isotropic path loss of mmWave propagation, they require a high MIMO-gain, which potentially requires a high number of MIMO-elements and their associated radio frequency (RF) chains. Naturally, this leads to high hardware cost and high energy consumption. Given their ability to reduce the number of RF chains, mmWave massive MIMO solutions relying on lens antenna arrays (LAA) have become popular [2]-[4]. By exploiting the energy-focusing capability of lens, the mmWave signals arriving from different directions may be focused on

W. Shen, L. Dai, Y. Li, and Z. Wang are with the Department of Electronic Engineering, Tsinghua University, Beijing 100084, China (E-mails: swq13@mails.tsinghua.edu.cn; \{daill, lyee, zcwang\}@tsinghua.edu.cn).

L. Hanzo is with the Department of Electronics and Computer Science, University of Southampton, Southampton SO17 1BJ, UK (e-mail: lh@ecs.soton.ac.uk).

This work was supported by the National Natural Science Foundation of China for Outstanding Young Scholars (Grant No. 61722109), the National Natural Science Foundation of China (Grant No. 61571270), and the Royal Academy of Engineering under the UK-China Industry Academia Partnership Programme Scheme (Grant No. UK-CIAPP $\backslash 49$ ). L. Hanzo would also like to acknowledge the financial support of the ERC Advanced Fellow Grant. different positions on the antenna array, so that the traditional spatial channel is transformed into the so-called beamspace channel. Due to the severe signal attenuation of mmWave frequencies, the number of dominant channel paths is limited in mmWave communication systems [5]. As a result, the beamspace channel created by the lens is sparse. Consequently, we can use a beam selector to select only a small number of energy-focusing beams, and reduce both the MIMO dimension and the number of RF chains.

As a benefit of beam selection, a high data rate can be achieved by performing downlink transmit-precoding (TPC) at the base station (BS) based on the reduced-dimensional equivalent channel. In frequency division duplexing (FDD) systems, the equivalent channel has to be signalled back to the BS via a feedback channel based on a dedicated codebook. However, the conventional random vector quantization (RVQ)-based codebook design [6], [7] is not applicable to mmWave massive MIMO systems relying on LAAs, since the equivalent channel vector is not Rayleigh distributed. The specific codebook based on the channel statistics of correlated channels [8] is not applicable either to mmWave massive MIMO systems using LAAs, since signalling the statistics of the equivalent channel back to the BS would impose an excessive overhead. To the best of our knowledge, there is no dedicated codebook design for channel feedback in mmWave massive MIMO systems relying on LAAs.

Hence in this paper, we propose a reduced-dimensional subspace codebook (RDSC) to fill this gap ${ }^{1}$. Specifically, we firstly exploit the recently proposed concept of angle coherence time to generate the large-dimensional vectors in the channel subspace, when the number of BS antennas $M$ tends to infinity, which mainly depends on the angles-of-departure (AoDs) of the dominant channel paths. Then, based on these vectors in the channel subspace, we design the RDSC by considering both the lens and the beam selector. Finally, the equivalent channel vector can be quantized using the proposed RDSC and fed back to the BS. Moreover, we also provide the mathematical analysis of the proposed RDSC and show that its feedback overhead is proportional to the reduced number of dominant paths per user, which is low.

Notation: Lower-case and upper-case boldface letters denote vectors and matrices, respectively. $(\cdot)^{\mathrm{T}},(\cdot)^{\mathrm{H}}$, and $(\cdot)^{-1}$ denote the transpose, conjugate transpose, and inverse of a matrix, respectively. $\boldsymbol{\Phi}^{\dagger}=\boldsymbol{\Phi}\left(\boldsymbol{\Phi}^{\mathrm{H}} \boldsymbol{\Phi}\right)^{-1}$ is the Moore-Penrose pseudo-

\footnotetext{
${ }^{1}$ Simulation codes are provided to reproduce the results presented in this paper: http://oa.ee.tsinghua.edu.cn/dailinglong/publications/publications.html.
} 
inverse. $\|\mathbf{h}\|$ and $|s|$ are the norm of a vector and the absolute value of a scalar, respectively. $\measuredangle(\mathbf{x}, \mathbf{y})$ is the angle between two vectors $\mathbf{x}$ and $\mathbf{y}$, and $\sin ^{2}(\measuredangle(\mathbf{x}, \mathbf{y}))=1-\frac{\left|\mathbf{x}^{H} \mathbf{y}\right|^{2}}{\|\mathbf{x}\|^{2}\|\mathbf{y}\|^{2}}$. $\mathrm{E}[\cdot]$ denotes the expectation operator. Finally, $\mathbf{I}_{P}$ denotes the identity matrix of size $P \times P$.

\section{System Model}

We commence by briefly introducing the typical downlink mmWave channel model. Then, we define the equivalent channel of mmWave massive MIMO systems using LAAs, which should be fed back to the BS for TPC.

\section{A. Downlink mmWave channel model}

We consider a mmWave massive MIMO system using $M$ antennas at the BS and $K$ single-antenna users $(M \gg K)$. For the widely used ray-based mmWave channel model of [1], the downlink channel vector $\mathbf{h}_{k} \in \mathbb{C}^{M \times 1}$ between the BS antennas and the $k$-th user can be described as

$$
\mathbf{h}_{k}=\sum_{i}^{P_{k}} g_{k, i} \mathbf{a}\left(\psi_{k, i}\right),
$$

where $P_{k}$ is the number of dominant paths of the $k$-th user, $g_{k, i}$ is the complex gain of the $i$-th path of the $k$-th user, which is identically and independently distributed (IID) with zero mean and unit variance ${ }^{2}$, and $\mathbf{a}\left(\psi_{k, i}\right) \in \mathbb{C}^{M \times 1}$ is the steering vector of the $i$-th path of the $k$-th user. We consider the widely used uniform linear array (ULA) ${ }^{3}$ at the BS, so $\mathbf{a}\left(\psi_{k, i}\right)$ can be expressed as [1]

$$
\mathbf{a}\left(\psi_{k, i}\right)=\frac{1}{\sqrt{M}}\left[1, e^{-j 2 \pi \psi_{k, i}}, \cdots, e^{-j 2 \pi \psi_{k, i}(M-1)}\right]^{\mathrm{T}},
$$

where $\psi_{k, i}=\frac{d}{\lambda} \sin \left(\theta_{k, i}\right)$ with $\theta_{k, i}$ denotes the AoD of the $i$-th path of the $k$-th user, $d$ denoting the BS antenna spacing, and $\lambda$ denoting the wavelength of the carrier frequency. Then we rewrite (1) in matrix form as

$$
\mathbf{h}_{k}=\mathbf{A}_{k} \mathbf{g}_{k},
$$

where $\mathbf{g}_{k}=\left[g_{k, 1}, g_{k, 2}, \cdots, g_{k, P_{k}}\right]^{\mathrm{T}} \in \mathbb{C}^{P_{k} \times 1}$, and $\mathbf{A}_{k}=$ $\left[\mathbf{a}\left(\psi_{k, 1}\right), \mathbf{a}\left(\psi_{k, 2}\right), \cdots, \mathbf{a}\left(\psi_{k, P_{k}}\right)\right] \in \mathbb{C}^{M \times P_{k}}$.

\section{B. Equivalent channel in LAA-aided mmWave systems}

The spatial channel $\mathbf{h}_{k}$ in (3) can be transformed into the beamspace channel by a carefully designed lens [2]-[4], as shown in Fig. 1. A lens is invoked before a ULA of antennas. This combination of a lens and a ULA of antennas is referred to as a LAA. Specifically, the signals arriving from different directions will be focused on different positions of a ULA of antennas after a lens. Such a lens can be characterized by a spatial-domain discrete Fourier transform (DFT) matrix $\mathbf{U}=$

\footnotetext{
${ }^{2}$ If large-scale fading coefficients are considered, which are not IID, the proposed RDSC can be appropriately modified according to the large-scale fading coefficients.

${ }^{3}$ Although a ULA is assumed in this paper, the proposed codebook can be readily extended to other antenna arrays, such as the uniform planar array [2].
}

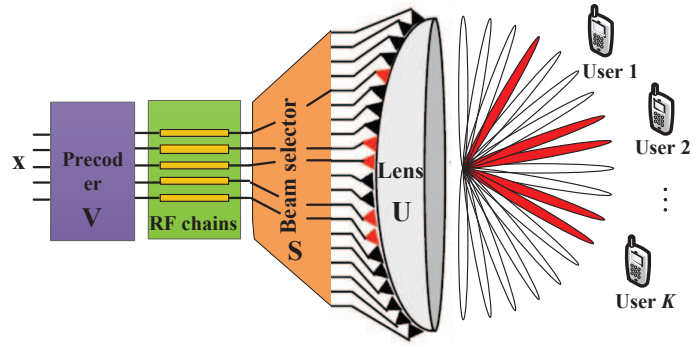

Fig. 1. Illustration of the mmWave massive MIMO systems relying on LAA.

$[\mathbf{a}(0), \mathbf{a}(\delta), \cdots, \mathbf{a}(\delta(M-1))]^{\mathrm{H}}$ with $\delta=\frac{1}{M}$ [2]. Therefore, the received signal $y_{k}$ at the $k$-th user can be expressed as

$$
y_{k}=\sqrt{\frac{\gamma}{K}} \mathbf{h}_{k}^{\mathrm{H}} \mathbf{U}^{\mathrm{H}} \mathbf{S} \mathbf{V} \mathbf{x}+n_{k}=\sqrt{\frac{\gamma}{K}}\left(\mathbf{h}_{k}^{\mathrm{b}}\right)^{\mathrm{H}} \mathbf{S} \mathbf{V} \mathbf{x}+n_{k},
$$

where $\gamma$ is the transmit power, $\mathbf{x}=\left[x_{1}, x_{2}, \cdots, x_{K}\right]^{\mathrm{T}} \in$ $\mathbb{C}^{K \times 1}$ is the data vector with normalized power $\mathrm{E}\left[\mathbf{x x}^{\mathrm{H}}\right]=\mathbf{I}_{K}$, and the beamspace channel vector $\mathbf{h}_{k}^{\mathrm{b}} \in \mathbb{C}^{M \times 1}$ can be expressed as

$$
\mathbf{h}_{k}^{\mathrm{b}}=\mathbf{U h}_{k},
$$

where the elements of $\mathbf{h}_{k}^{\mathrm{b}}$ are the channel coefficients associated with $M$ orthogonal beams. Since the channel is composed of $P_{k}$ dominant paths, there are only $P_{k}$ dominant elements in $\mathbf{h}_{k}^{b}$ [2]. $\mathbf{S} \in \mathbb{C}^{M \times N_{\mathrm{RF}}}$ is the beam selector, with $N_{\mathrm{RF}}$ denoting the number of RF chains at the BS. Each column of $\mathbf{S}$ has one non-zero element ' 1 ', whose location is different in the different columns. Furthermore, $n_{k}$ is the complex Gaussian noise at the $k$-th user with zero mean and unit variance.

We define the reduced-dimensional equivalent channel $\mathbf{h}_{k}^{\mathrm{e}} \in$ $\mathbb{C}^{N_{\mathrm{RF}} \times 1}$ as

$$
\mathbf{h}_{k}^{\mathrm{e}}=\mathbf{S}^{\mathrm{H}} \mathbf{h}_{k}^{\mathrm{b}} .
$$

The equivalent multi-user MIMO channel matrix can be expressed as $\mathbf{H}^{\mathrm{e}}=\left[\mathbf{h}_{1}^{\mathrm{e}}, \mathbf{h}_{2}^{\mathrm{e}}, \cdots, \mathbf{h}_{K}^{\mathrm{e}}\right] \in \mathbb{C}^{N_{\mathrm{RF}} \times K}$. Thus (4) can be rewritten as

$$
y_{k}=\sqrt{\frac{\gamma}{K}}\left(\mathbf{h}_{k}^{\mathrm{e}}\right)^{\mathrm{H}} \mathbf{V} \mathbf{x}+n_{k},
$$

where $\mathbf{V}=\left[\mathbf{v}_{1}, \mathbf{v}_{2}, \cdots, \mathbf{v}_{K}\right] \in \mathbb{C}^{N_{\mathrm{RF}} \times K}$ is the zero-forcing (ZF) precoding matrix with $\mathbf{v}_{i}=\frac{\left(\hat{\mathbf{H}}^{\mathrm{e}}\right)^{\dagger}(:, i)}{\left\|\left(\hat{\mathbf{H}}^{\mathrm{e}}\right)^{\dagger}(:, i)\right\|}$, where $\hat{\mathbf{H}}^{\mathrm{e}}=$ $\left[\hat{\mathbf{h}}_{1}^{\mathrm{e}}, \hat{\mathbf{h}}_{2}^{\mathrm{e}}, \cdots, \hat{\mathbf{h}}_{K}^{\mathrm{e}}\right] \in \mathbb{C}^{N_{\mathrm{RF}} \times K}$ and $\hat{\mathbf{h}}_{k}^{\mathrm{e}}$ is the fedback equivalent channel, which is determined based on the RDSC proposed in the next section.

\section{Proposed Reduced-Dimensional Subspace CODEBOOK}

In this section, we propose the RDSC for quantizing the equivalent channel. Then, we provide the performance analysis of the proposed RDSC.

\section{A. Proposed RDSC}

Firstly, we exploit the recently proposed concept of angle coherence time [9], [10] defined as the period, within which the AoDs remain near-constant. During the angle coherence time, the channel vector $\mathbf{h}_{k}=\mathbf{A}_{k} \mathbf{g}_{k}$ in (3) is distributed in 
the column space of $\mathbf{A}_{k} \in \mathbb{C}^{M \times P_{k}}$, which is termed as the channel subspace, determined by $P_{k}(\ll M)$ AoDs. Therefore, we first generate the large-dimensional vectors in the channel subspace as follows

$$
\mathbf{c}_{k, i}=\mathbf{A}_{k} \mathbf{w}_{k, i},
$$

where $\mathbf{w}_{k, i} \in \mathbb{C}^{P_{k} \times 1}$ is isotropically distributed with a unitnorm $\left(i=1,2, \cdots, 2^{B}\right.$, with $B$ being the number of feedback bits). $\mathbf{A}_{k}$ is determined by the AoDs of the $k$-th user, which can be determined at a low average overhead [11], since the angle coherence time is much longer than the traditional channel coherence time.

Then, based on the large-dimensional vector $\mathbf{c}_{k, i}$, we propose the RDSC $\mathcal{D}_{k}=\left\{\mathbf{d}_{k, 1}, \mathbf{d}_{k, 2}, \cdots, \mathbf{d}_{k, 2^{B}}\right\}$, where $\mathbf{d}_{k, i} \in$ $\mathbb{C}^{N_{\mathrm{RF}} \times 1}$ can be expressed as

$$
\mathbf{d}_{k, i}=\mathbf{S}^{\mathrm{H}} \mathbf{U} \mathbf{c}_{k, i}
$$

where the beam selector $\mathbf{S}$ can be designed based on the AoDs during the angle coherence time [12]. To satisfy the unit-norm requirement of the codeword $\mathbf{d}_{k, i}$, it should be normalized as

$$
\mathbf{d}_{k, i}=\frac{\mathbf{d}_{k, i}}{\left\|\mathbf{d}_{k, i}\right\|} .
$$

Finally, the $k$-th user will quantize the equivalent channel $\mathbf{h}_{k}^{\mathrm{e}}$ based on the proposed RDSC by finding $\mathbf{d}_{k, F_{k}}$ that is closest to $\mathbf{h}_{k}^{\mathrm{e}}$, where the index $F_{k}$ is computed as

$$
F_{k}=\underset{i \in\left\{1,2, \cdots, 2^{B}\right\}}{\arg \min } \sin ^{2}\left(\measuredangle\left(\tilde{\mathbf{h}}_{k}^{\mathrm{e}}, \mathbf{d}_{k, i}\right)\right),
$$

where $\tilde{\mathbf{h}}_{k}^{\mathrm{e}}=\frac{\mathbf{h}_{k}^{\mathrm{e}}}{\left\|\mathbf{h}_{k}^{\mathrm{e}}\right\|}$ represents the direction of the equivalent channel. Since the magnitude of the equivalent channel $\left\|\mathbf{h}_{k}^{\mathrm{e}}\right\|$ is just a scalar, we follow the widely used assumption that this magnitude $\left\|\mathbf{h}_{k}^{\mathrm{e}}\right\|$ can be fed back to the BS perfectly [6], [7], [9]. The codebook index $F_{k}$ can be fed back using $B$ bits. As a result, the $\mathrm{BS}$ receives the equivalent channel vector in the uplink as $\hat{\mathbf{h}}_{k}^{\mathrm{e}}=\left\|\mathbf{h}_{k}^{\mathrm{e}}\right\| \mathbf{d}_{k, F_{k}}$. In the next subsection, we will analyse the performance of the proposed RDSC.

\section{B. Performance analysis of the proposed RDSC}

We can rewrite (7) as

$$
y_{k}=\sqrt{\frac{\gamma}{K}}\left(\mathbf{h}_{k}^{\mathrm{e}}\right)^{\mathrm{H}} \mathbf{v}_{k} x_{k}+\sqrt{\frac{\gamma}{K}} \sum_{i=1, i \neq k}^{K}\left(\mathbf{h}_{k}^{\mathrm{e}}\right)^{\mathrm{H}} \mathbf{v}_{i} x_{i}+n_{k},
$$

Thus, the per-user rate of the mmWave massive MIMO systems relying on LAAs and ZF TPC becomes:

$$
R=\mathrm{E}\left[\log _{2}\left(1+\frac{\frac{\gamma}{K}\left|\left(\mathbf{h}_{k}^{\mathrm{e}}\right)^{\mathrm{H}} \mathbf{v}_{k}\right|^{2}}{1+\frac{\gamma}{K} \sum_{i=1, i \neq k}^{K}\left|\left(\mathbf{h}_{k}^{\mathrm{e}}\right)^{\mathrm{H}} \mathbf{v}_{i}\right|^{2}}\right)\right],
$$

where $R$ in (13) is obtained based on the fed-back equivalent channel $\hat{\mathbf{H}}^{\mathrm{e}}$. For the ideal case of a perfect equivalent channel $\mathbf{H}^{\mathrm{e}}$ at the $\mathrm{BS}$, the $\mathrm{ZF}$ precoding can be performed based on $\mathbf{H}^{\mathrm{e}}$. In this ideal case, we can obtain the per-user rate as

$$
R_{\text {ideal }}=\mathrm{E}\left[\log _{2}\left(1+\frac{\gamma}{K}\left|\left(\mathbf{h}_{k}^{\mathrm{e}}\right)^{\mathrm{H}} \mathbf{v}_{\text {ideal }, k}\right|^{2}\right)\right],
$$

where $\mathbf{v}_{\text {ideal }, i}$ is obtained as the normalized $i$-th column of $\left(\mathbf{H}^{\mathrm{e}}\right)^{\dagger}$. The rate-loss $\Delta R(\gamma)$ is defined as

$$
\Delta R(\gamma)=R_{\text {ideal }}-R
$$

which can be upper-bounded following the results of [6]

$$
\Delta R(\gamma) \leq \log _{2}\left(1+\frac{\gamma(K-1)}{K} \mathrm{E}\left[\left\|\mathbf{h}_{k}^{\mathrm{e}}\right\|^{2}\right] \mathrm{E}\left[\sin ^{2}\left(\measuredangle\left(\tilde{\mathbf{h}}_{k}^{\mathrm{e}}, \hat{\mathbf{h}}_{k}^{\mathrm{e}}\right)\right)\right]\right)
$$

We can observe from (16) that the rate-loss $\Delta R(\gamma)$ is dominanted by the quantization error $\mathrm{E}\left[\sin ^{2}\left(\measuredangle\left(\tilde{\mathbf{h}}_{k}^{\mathrm{e}}, \hat{\mathbf{h}}_{k}^{\mathrm{e}}\right)\right)\right]$. Next, we will discuss the quantization error, which is different from that in [6] and [9] due to the specific distribution of the equivalent channel $\mathbf{h}_{k}^{\mathrm{e}}$. In the rest of this paper, we can omit the subscript $k$ without loss of generality, where $\mathbf{h}^{\mathrm{e}}, \mathbf{A}$, and $P$ denote the equivalent channel, the steering matrix, and the number of dominant paths per user, respectively. Before discussing the quantization error, we will prove the Lemma 1 and Lemma 2, which will be useful for the analysis of the quantization error in Lemma 3.

Lemma 1: In the large-dimensional regime, i.e. when the number of BS antennas $M$ tends to infinity, we have $\mathbf{T}^{\mathrm{H}} \mathbf{T}=$ $\mathbf{I}_{P}$ with $\mathbf{T}=\mathbf{S}^{\mathrm{H}} \mathbf{U A}$.

Proof: We firstly calculate the $(i, j)$-th element of $\mathbf{U A}$, which is obtained as the product of the $i$-th row vector $\mathbf{a}(\delta(i-$ $1))^{\mathrm{H}}$ of $\mathbf{U}$ and the $j$-th column vector $\mathbf{a}\left(\psi_{j}\right)$ of $\mathbf{A}$. In the large-dimensional regime, due to the orthogonality between $\mathbf{a}(\delta(i-1))$ and $\mathbf{a}\left(\psi_{j}\right)\left(\forall \psi_{j} \neq \delta(i-1)\right)$ [9], UA has one nonzero element " 1 " in each column, whose location is different in different columns. Note that the beam selector $\mathbf{S}$ is designed to choose $N_{\mathrm{RF}}$ rows of $\mathbf{U A}$ which includes non-zero elements and $N_{\mathrm{RF}}$ is usually much larger than $P$ (e.g., $N_{\mathrm{RF}}=P K$ [2]). Thus, $\mathbf{T} \in \mathbb{C}^{N_{\mathrm{RF}} \times P}$ has one non-zero element "1" in each column, whose location is different in different columns. Therefore, we can obtain $\mathbf{T}^{\mathrm{H}} \mathbf{T}=\mathbf{I}_{P}$.

Lemma 2: $\left\|\mathbf{h}^{\mathrm{e}}\right\|=\left\|\mathbf{S}^{\mathrm{H}} \mathbf{U A g}\right\|=\|\mathbf{g}\|$.

Proof: Combining (3), (5) and (6), we have

$$
\mathbf{h}^{\mathrm{e}}=\mathbf{S}^{\mathrm{H}} \mathbf{U A g}=\mathbf{T g} .
$$

By invoking Lemma 1, we have

$$
\left\|\mathbf{h}^{\mathrm{e}}\right\|^{2}=\mathbf{g}^{\mathrm{H}} \mathbf{T}^{\mathrm{H}} \mathbf{T g}=\|\mathbf{g}\|^{2} .
$$

Based on Lemma 1 and Lemma 2 above, we now derive an upper bound of the quantization error in (16) in the following Lemma 3.

Lemma 3: The quantization error $\mathrm{E}\left[\sin ^{2}\left(\measuredangle\left(\tilde{\mathbf{h}}^{\mathrm{e}}, \hat{\mathbf{h}}^{\mathrm{e}}\right)\right)\right]$ can be upper bounded as

$$
\mathrm{E}\left[\sin ^{2}\left(\measuredangle\left(\tilde{\mathbf{h}}^{\mathrm{e}}, \hat{\mathbf{h}}^{\mathrm{e}}\right)\right)\right]<2^{-\frac{B}{P-1}} .
$$
we have

Proof: Since $\left\|\tilde{\mathbf{h}}^{\mathrm{e}}\right\|=1, \hat{\mathbf{h}}^{\mathrm{e}}=\left\|\mathbf{h}^{\mathrm{e}}\right\| \mathbf{d}_{F}$, and $\left\|\mathbf{d}_{F}\right\|=1$,

$$
\mathrm{E}\left[\sin ^{2}\left(\measuredangle\left(\tilde{\mathbf{h}}^{\mathrm{e}}, \hat{\mathbf{h}}^{\mathrm{e}}\right)\right)\right]=1-\mathrm{E}\left[\left|\mathbf{d}_{F}^{\mathrm{H}} \tilde{\mathbf{h}}^{\mathrm{e}}\right|^{2}\right] .
$$


Following Lemma 2, we have $\tilde{\mathbf{h}}^{\mathrm{e}}=\frac{\mathbf{T g}}{\left\|\mathbf{h}^{\|}\right\|}=\mathbf{T} \tilde{\mathbf{g}}$, where $\tilde{\mathbf{g}}=$ $\frac{\mathbf{g}}{\|\mathbf{g}\|}$. Considering (9) and using $\left\|\mathbf{w}_{F}\right\|=1$, we have $\mathbf{d}_{F}=$ $\mathbf{T w}_{F}$. Therefore,

$$
\mathrm{E}\left[\left|\mathbf{d}_{F}^{\mathrm{H}} \tilde{\mathbf{h}}^{\mathrm{e}}\right|^{2}\right]=\mathrm{E}\left[\left|\mathbf{w}_{F}^{\mathrm{H}} \mathbf{T}^{\mathrm{H}} \mathbf{T} \tilde{\mathbf{g}}\right|^{2}\right] \stackrel{(a)}{=} \mathrm{E}\left[\left|\mathbf{w}_{F}^{\mathrm{H}} \tilde{\mathbf{g}}\right|^{2}\right],
$$

where (a) follows from the result $\mathbf{T}^{\mathrm{H}} \mathbf{T}=\mathbf{I}_{P}$ in Lemma 1. Since $\mathbf{w}_{F}$ and $\tilde{\mathbf{g}}$ are isotropically distributed $P \times 1$ vectors, $\mathrm{E}\left[\left|\mathbf{w}_{F}^{\mathrm{H}} \tilde{\mathbf{g}}\right|^{2}\right]$ can be lower-bounded as [6]:

$$
\mathrm{E}\left[\left|\mathbf{w}_{F}^{\mathrm{H}} \tilde{\mathbf{g}}\right|^{2}\right]>1-2^{-\frac{B}{P-1}} .
$$

Combining (20), (21), and (22), we can obtain (19).

Finally, by substituting (19) into (16), we have

$$
\Delta R(\gamma) \leq \log _{2}\left(1+\frac{\gamma(K-1)}{K} \mathrm{E}\left[\left\|\mathbf{h}^{\mathrm{e}}\right\|^{2}\right] 2^{-\frac{B}{P-1}}\right) .
$$

Let $\Delta R(\gamma) \leq \log _{2}\left(1+\frac{\gamma(K-1)}{K} \mathrm{E}\left[\left\|\mathbf{h}^{\mathrm{e}}\right\|^{2}\right] 2^{-\frac{B}{P-1}}\right) \leq 1$ bp$\mathrm{s} / \mathrm{Hz}$, the number of feedback bits $B$ should be

$$
B \geq \frac{P-1}{3} \mathrm{SNR}+(P-1) \log _{2}(K-1),
$$

where $\mathrm{SNR}=10 \log _{10} \frac{\gamma}{K} \mathrm{E}\left[\left\|\mathbf{h}^{\mathrm{e}}\right\|^{2}\right]$ is the signal-to-noise-ratio (SNR) at the receiver. We can observe from (24) that the slope of the required number of feedback bits $B$ is $P-1$ when the SNR increases. In other words, the required number of feedback bits $B$ only scales linearly with $P-1$ to maintain a constant rate gap. Since $P \ll N_{\mathrm{RF}}$, the proposed RDSC requires much lower feedback overhead than the conventional RVQ-based codebook, where the number of feedback bits scales linearly with $N_{\mathrm{RF}}$. In the next section, we will verify the analytical results by simulations.

\section{Simulation Results}

The key system parameters are set as follows: $M=128$, $N_{\mathrm{RF}}=24, K=8$, and $P=3$. The AoDs are randomly chosen from the uniform distribution $\mathcal{U}\left[-\frac{1}{2} \pi, \frac{1}{2} \pi\right]$. The number of feedback bits is set to $B=\frac{P-1}{3}$ SNR $+(P-1) \log _{2}(K-1)$. Fig. 2 shows the per-user rates of the ideal case associated with a perfect equivalent channel at the BS and the practical case with channel feedback, where the proposed RDSC, the conventional RVQ-based codebook, and direct feedback of path gains are used. We observe from Fig. 2 that the rate-gap between the ideal case with perfect equivalent channel and the practical case with the proposed RDSC remains constant, when the SNR increases, which is consistent with the theoretical analysis of Section III-B. Additionally, we also observe that the proposed RDSC outperforms the conventional RVQ-based codebook and direct feedback of path gains.

\section{CONCLUSIONS}

In this paper, we have proposed a beneficial codebook design for channel feedback in mmWave massive MIMO systems relying on LAAs. Specifically, we observed that the equivalent channel in mmWave massive MIMO systems using LAAs is distributed in a reduced-dimensional channel subspace. Thus, we firstly proposed to generate the high-dimensional vectors in the channel subspace. Then, by considering the lens and beam

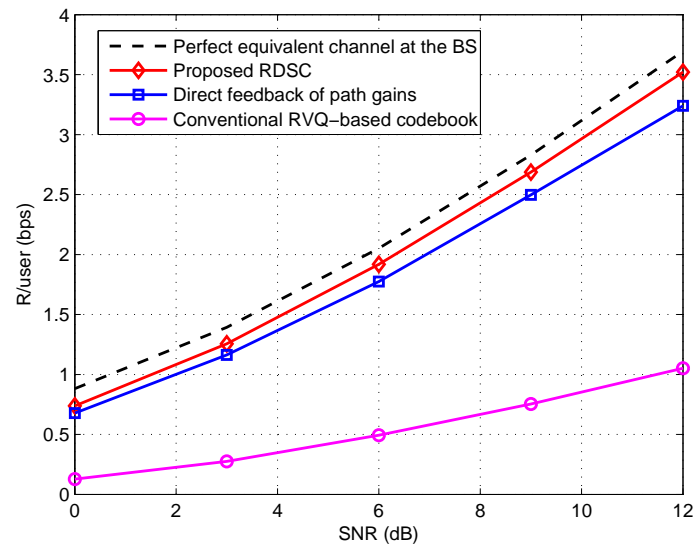

Fig. 2. Per-user rate comparison between the ideal case with perfect equivalent channel at the BS and the practical case with channel feedback.

selector, we proposed our RDSC to quantize the equivalent channel, which was fed back to the BS. Moreover, we provided the performance analysis of the proposed RDSC and showed that the feedback overhead using the proposed RDSC is only proportional to the number of dominant paths per user, which is small. Our simulation results verify the analytical results.

\section{REFERENCES}

[1] R. W. Heath, N. Gonzalez-Prelcic, S. Rangan, W. Roh, and A. Sayeed, "An overview of signal processing techniques for millimeter wave MIMO systems," IEEE J. Sel. Top. Signal Process., vol. 10, no. 3, pp. 436-453, Apr. 2016.

[2] J. Brady, N. Behdad, and A. M. Sayeed, "Beamspace MIMO for millimeter-wave communications: System architecture, modeling, analysis, and measurements," IEEE Trans. Antennas Propag., vol. 61, no. 7, pp. 3814-3827, Jul. 2013.

[3] Y. Zeng, R. Zhang, and Z. N. Chen, "Electromagnetic lens-focusing antenna enabled massive MIMO: Performance improvement and cost reduction," IEEE J. Sel. Areas Commun., vol. 32, no. 6, pp. 1194-1206, Jun. 2014.

[4] Y. Zeng and R. Zhang, "Millimeter wave MIMO with lens antenna array: A new path division multiplexing paradigm," IEEE Trans. Commun., vol. 64, no. 4, pp. 1557-1571, Apr. 2016.

[5] T. S. Rappaport, S. Sun, R. Mayzus, H. Zhao, Y. Azar, K. Wang, G. N. Wong, J. K. Schulz, M. Samimi, and F. Gutierrez, "Millimeter wave mobile communications for 5G cellular: It will work!" IEEE Access, vol. 1, pp. 335-349, May 2013.

[6] N. Jindal, "MIMO broadcast channels with finite-rate feedback," IEEE Trans. Inf. Theory, vol. 52, no. 11, pp. 5045-5060, Nov. 2006.

[7] A. Alkhateeb, G. Leus, and R. W. Heath, "Limited feedback hybrid precoding for multi-user millimeter wave systems," IEEE Trans. Wireless Commun., vol. 14, no. 11, pp. 6481-6494, Nov. 2015.

[8] D. J. Love and R. W. Heath, "Limited feedback diversity techniques for correlated channels," IEEE Trans. Veh. Technol., vol. 55, no. 2, pp. 718-722, Mar. 2006.

[9] W. Shen, L. Dai, G. Gui, Z. Wang, R. W. Heath, and F. Adachi, "AoDadaptive subspace codebook for channel feedback in FDD massive MIMO systems," in Proc. IEEE International Conference on Communications (IEEE ICC'17), May 2017, pp. 1-6.

[10] V. Va, J. Choi, and R. Heath, "The impact of beamwidth on temporal channel variation in vehicular channels and its implications," IEEE Trans. Veh. Technol., vol. 66, no. 6, pp. 5014-5029, Jun. 2017.

[11] X. Gao, L. Dai, S. Han, C. L. I, and X. Wang, "Reliable beamspace channel estimation for millimeter-wave massive MIMO systems with lens antenna array," IEEE Trans. Wireless Commun., vol. 16, no. 9, pp. 6010-6021, Sep. 2017.

[12] X. Gao, L. Dai, Z. Chen, Z. Wang, and Z. Zhang, "Near-optimal beam selection for beamspace mmwave massive MIMO systems," IEEE Commun. Lett., vol. 20, no. 5, pp. 1054-1057, May 2016. 